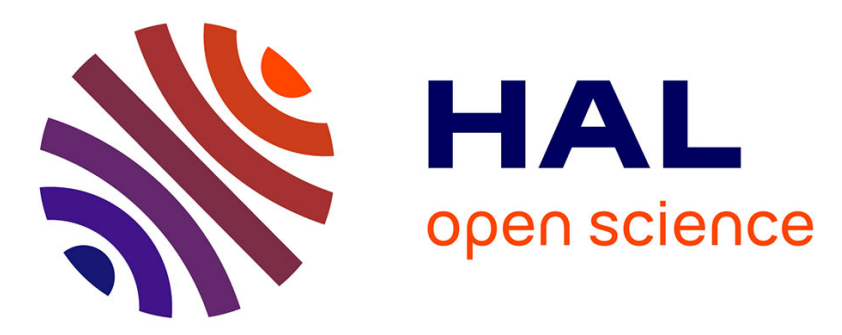

\title{
Frictionless contact of elastic bodies by finite element method and mathematical programming technique
}

\author{
Nguyen Dang Hung, Géry De Saxcé
}

\section{To cite this version:}

Nguyen Dang Hung, Géry De Saxcé. Frictionless contact of elastic bodies by finite element method and mathematical programming technique. Computers \& Structures, 1980, 11 (1-2), pp.55-67. 10.1016/0045-7949(80)90146-7 . hal-01828018

\section{HAL Id: hal-01828018 https://hal.science/hal-01828018}

Submitted on 20 Sep 2018

HAL is a multi-disciplinary open access archive for the deposit and dissemination of scientific research documents, whether they are published or not. The documents may come from teaching and research institutions in France or abroad, or from public or private research centers.
L'archive ouverte pluridisciplinaire HAL, est destinée au dépôt et à la diffusion de documents scientifiques de niveau recherche, publiés ou non, émanant des établissements d'enseignement et de recherche français ou étrangers, des laboratoires publics ou privés. 


\title{
FRICTIONLESS CONTACT OF ELASTIC BODIES BY FINITE ELEMENT METHOD AND MATHEMATICAL PROGRAMMING TECHNIQUE
}

\author{
NGUYen DANg Hungt and Géry de SAXCE‡ \\ Department of Structural Mechanics and Stability of Constructions, University of Liżge, Belgium
}

(Received 13 June 1979)

\begin{abstract}
The work presents an analysis of the contact problems between elastic bodies without friction. Extended variational principles in the contact problems constitute the theoretical foundations of the analysis. In fact, kinematically admissible displacement fields are subjected to the non-interpenetration conditions so that the contact problem being equivalent to a minimization of the potential energy with contraints on the displacements may be reduced to a particular case of the mathematical programming techniques. Applications are limited to plane state of stress or strain cases where small deformations are considered. The geometrical space is discretized into finite elements, namely the 12 degree of freedom hybrid triangles or the 16 degrees of freedom isoparametric bi-linear quadrangle elements. A special procedure of linearization is adopted to use the quadratic programming algorithm already prepared for large scale problems.

In these conditions, a computer program has been written in FORTRAN IV H and the results obtained from this present good agreement comparing to analytical solutions or other numerical solution in the literature. Finally, theoretical efforts have been performed to generalize the method by introducing a concept of fictitious locked material which represents the assumed contact space between the two bodies.

A general and new condition of non-interpenetration is proposed for general cases of two elastic solids in contact.
\end{abstract}

\section{NOMENCLATURE}

$x_{i} \quad$ Lagrangian coordinates

$\zeta_{i}$ Eulerian coordinates

$V, V, V_{B}$ geometric domains of elastic bodies

$\Gamma_{\theta}, \Gamma_{A A}, \Gamma_{\theta B}$ portion of boundary of $V, V_{A}, V_{B}$ where $\bar{t}_{t}$ are prescribed

î, $\eta_{i}$ normal vector defined on the boundaries

$\boldsymbol{\Omega}$ rigid domain

$\partial \Omega$ boundary of $\Omega$

$\varepsilon_{i j}, \varepsilon$ strain tensor, strain field

$U\left(\varepsilon_{i j}\right)$ density of the strain energy

$\sigma_{i j}, \sigma$ stress tensor, stress field

$\tau_{i i}$ stress tensor (Piola) (Euler-cauchy)

$J_{i}$ body forces

$\bar{t}_{i}$ tractions prescribed on the boundaries

$q$ nodal displacement vector

g nodal generalized force vector

global stifiness matrix

D total potential strain energy

Ф* modified total potential energy

$\Phi_{c}$ contact potential

$\phi_{c}$ dislocation potential

II contact constraint function

$\lambda$ Lagrange's parameter

$\omega$ slack variable

$A$ constraint matrix

$D_{i}, \nabla$ Lagrangian gradient operator

$\alpha_{i j}$ displacement gradient

$\left[J_{i j}\right]$ Jacobian matrix

$J$ Jacobian, determinant of $\left[J_{i j}\right]$

$I_{i j}$ minor of element $J_{i j}$ in $\left[J_{i j}\right]$

\section{INTRODUCTION}

The problem of contact of elastic bodies even in small deformations is a nonlinear problem because of the complicating factor: the presence of the unknown surface contact. One may find in the literature two distinct ways of numerical approach for this problem; the iterative method; and the direct method.

The first method consists of calculating the increment of loading and verifying the contact condition at each step. Following authors may be cited among the adepts of this procedure. Chan and Tuba [1], Gaertner [2], Zienkiewicz and Francavilla [3], Fredriksson [4].

The second method consists of reducing the elastic contact problem to a special case of the mathematical programming techniques. By that way, Feng and Huang [5] have examined the contact problem of an inflated plane membrane. The variational forms of the contact problem is studied by Fremond [6] who has presented some numerical examples of contact between elastic bodies and rigid foundation. Panagiotopoulos [7] has generalized this approach to the inelastic foundation and presented some dual forms of the variational inequalities for the contact problem.

The present work belongs to the second type of formulation where appropriate linearization of contact condition is adopted. It is assumed that no friction between solid bodies exists so that the contact condition may be expressed uniquely in terms of displacements. It is supposed also that the deformation is small and the material obeys the linear elastic constitutive equations. In the numerical examples, we will be dealing only with plane strain or stress two dimensions problems. The generalization of the formulation to the three dimensions bodies would

† Maître de Conférences.

†Engineer Physician. 
present no major difficulties but a considerable increasing number of variables. This paper may be divided in two sections. In the first section, the contact between an elastic body and a rigid foundation is considered.

Good agreement numerical results obtained using the present finite element algorithm adopting this formulation and the super-element technique are illustrated.

In the second section, theoretical efforts are performed to generalize the formulation. We introduce a concept of representing the space separating the contacting surface as a fictitious perfectly locking material.

This concept is pointed out by adopting a new and general non-interpenetration condition which involves important commodity for the formulation of the contact problem of elastic bodies. It may be seen that in the particular case of rigid-elastic contact under small deformations assumption, the general noninterpenetration condition is reduced to the constraint condition of the case mentioned above.

\section{CONTACT OF AN ELASTIC BODY AND A RIGID FOUNDATION}

2.1 Kinematically admissible displacements in the sense of contact problem

Let $\Omega$ be the rigid foundation and $\partial \Omega$ its boundary. Let $V$ be the elastic body. Its boundary is composed of three portions: $\Gamma_{\sigma}$ where surface tractions $\bar{t}_{i}$ are prescribed, $\Gamma_{u}$ where displacements $\bar{u}_{i}$ are prescribed and $\Gamma_{c}$ where possible contact may happen (Fig. 1).

The initial configuration of the body $V$ is determined by a position vector $\mathrm{OP}=\mathbf{x}$. The components of which $x_{i}$ are the Lagrangian coordinates of the material point. Let $\mathbf{u}(\mathbf{x})$ be the displacement vector of the point $P$ under loading. The new position $P^{\prime}$ of $P$ after deformation is described by the new coordinates

$$
0 \mathbf{P}^{\prime}=\xi=\mathbf{x}+\mathbf{u}
$$

where the components of $\xi_{,} \xi_{i}$ are Eulerian coordinates of the material point $P^{\prime}$.
We assume that the surface of the foundation $c \Omega$ is regular so that it may be defined by the following equation:

$$
\operatorname{II}(\xi)=0 .
$$

In practice, eqn (2.2) defines only the possible contacting surface but not necessarily the whole surface $\partial \Omega$. By convention, we suppose that the region where $\operatorname{II}(\xi)$ is negative belongs to the foundation $\Omega$. The displacement field $\mathbf{u}$ is kinematically admissible in contact problem sense if it satisfied the following noninterpenetration condition

$$
\Gamma \mathrm{II}(\mathbf{x}+\mathbf{u}) \geqslant 0 .
$$

\subsection{Displacement variational principle}

The classical total potential energy of an elastic body $V$ subjected to imposed body forces $\bar{f}_{i}$ and prescribed traction $\bar{t}_{i}$ on $\Gamma_{\sigma}$ is

$\Phi\left(u_{i}\right)=\int U\left(\varepsilon_{i j}\right) \mathrm{d} V-\int f_{i} u_{i} \mathrm{~d} V-\int \bar{t}_{i} u_{i} \mathrm{~d} \Gamma_{\sigma}$

where $U$ is the density of strain energy.

$\varepsilon_{i j}$ is kinematically admissible strain field such that

$$
\varepsilon_{i j}=\frac{1}{2}\left(D_{i} u_{j}+D_{j} u_{i}\right)
$$

The contact problem [6] is reduced to a minimization of the functional (2.4) subjected to the constraint (2.3) with respect to arbitrary kinematically admissible displacement field $u_{i}$ minimized $\Phi\left(u_{i}\right)$.

$$
\begin{gathered}
u_{i} \in H_{\mathbf{K}} \\
H_{\mathrm{K}}=\left\{u_{i} \mid u_{i}=\bar{u}_{i} \text { on } \Gamma_{u}\right.
\end{gathered}
$$

and

$$
\left.\Pi\left(x_{i}+u_{i}\right) \geqslant 0 \text { on } \Gamma_{\epsilon}\right\}
$$

Now introducing a slack variable $\omega$ the inequality (2.3) may be substituted by the following equality:

$$
\Pi\left(x_{i}+u_{i}\right)-\omega^{2}=0
$$

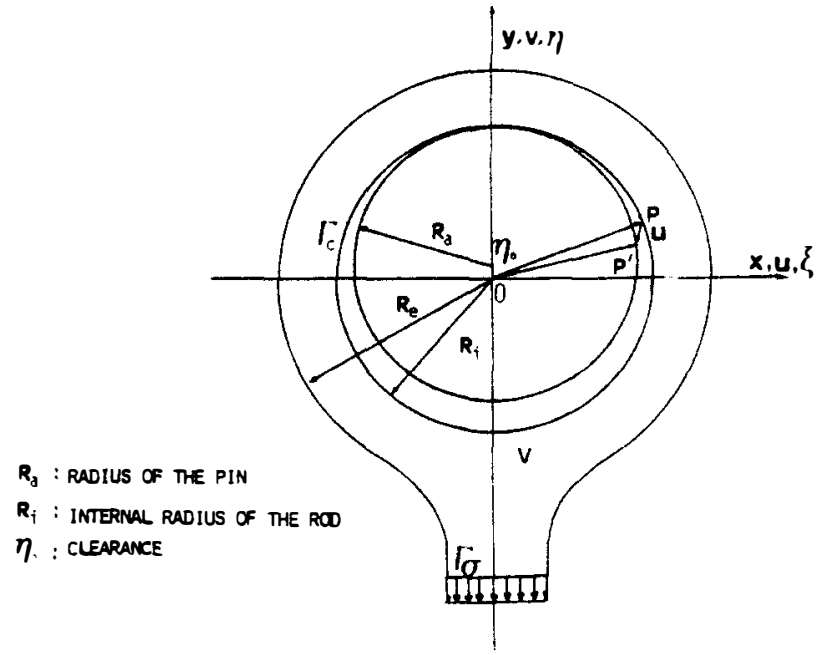

Fig. 1. Contact surface $\Gamma_{c}$ and external surface $\Gamma_{\sigma}$ contact between a pin and a piston rod 
and using a Lagrange's parameter $\lambda$ a relaxation of (2.7) may be performed by a dislocation potential according to Friedrich

$\phi\left(u_{i}, \lambda, \omega\right)=$

$$
-\int \lambda\left[\Pi\left(x_{i}+u_{i}\right)-\omega^{2}\right] \mathrm{d} \Gamma_{c}
$$

If a modified functional $\Phi^{*}$ is adopted such that

$$
\Phi^{*}\left(u_{i}, \lambda, \omega\right)=\Phi\left(u_{i}\right)+\phi\left(u_{i}, \lambda, \omega\right)
$$

the following variational problem is equivalent to (2.6):

$$
\delta \Phi^{*}\left(u_{i}, \lambda, \omega\right)=0
$$

with $u_{i}, H=\left\{u_{i} \mid u_{i}=\bar{u}_{i}\right.$ on $\left.\Gamma_{u}\right\}$.

There are no difficulties to see that the Euler-Lagrange equations of the problem (2.10) are

Internal equilibrium: $D_{j} \sigma_{i j}+f_{i}=0$

Surface equilibrium on $\Gamma_{j}: n_{j} \sigma_{i j}=\bar{t}_{i}$

Contact equilibrium on $\Gamma_{c}: n_{j} \sigma_{i j}=\lambda D_{i} \pi$

Displacement constraint on $\Gamma_{c}: \Pi\left(x_{i}+u_{i}\right)-\omega^{2}=0$

Complementarity condition on $\Gamma_{c}: \lambda \omega=0$.

It appears from (2.15) that if $\omega \neq 0$, then $\lambda=0$. There is no contact according to (2.14) and by (2.13) no contact pressure takes place. If $\omega=0$ then $\lambda>0$ and there is contact. So the complementarity condition (2.15) restores the Kuhn-Tucker optimality conditions. The Fig. 2 illustrates the situation discussed above. An interpretation of the Lagrange parameter $\lambda$ may be performed in the following way. There is no friction so the surface tractions must be normal to the contacting surface $\Gamma_{c}$
Consequently

$$
\lambda=\frac{\|t\|}{\|\nabla \Pi\|} .
$$

If one has chosen the normalized constraint function

$$
\Pi_{0}=\Pi /\|\nabla \Pi\|
$$

instead of $\Pi$, then $\lambda$ is identified exactly as the contact pressure.

\subsection{Finite element formulation}

Classical discretization using compatible finite element and displacement method leads to the following forms of total potential energy of the whole structure:

$$
\Phi=\frac{1}{2} q^{T} K q-g^{T} q
$$

where $q$ is nodal displacement vector $g$ is generalized force vector $\boldsymbol{K}$ is the stiffness matrix.

The finite element discretization of the contact potential

$$
\Phi_{c}=\int \lambda \Pi\left(x_{i}+u_{i}\right) d \Gamma_{c}
$$

may be realized in the following way.

Assuming the displacements are small. We may remain only the first order terms of the Taylor's expansion of the function $\Pi\left(x_{i}+u_{i}\right)$

$$
\Pi\left(x_{i}+u_{i}\right) \simeq \Pi\left(x_{i}\right)+D_{k} \Pi\left(x_{i}\right) u_{k}+0\left(u_{i} u_{k}\right)
$$

So that inequality (2.3) becomes

$$
D_{k} \Pi\left(x_{i}\right) u_{k} \geqslant-\Pi\left(x_{i}\right)
$$

$$
\mathbf{t}=\lambda \nabla \Pi
$$

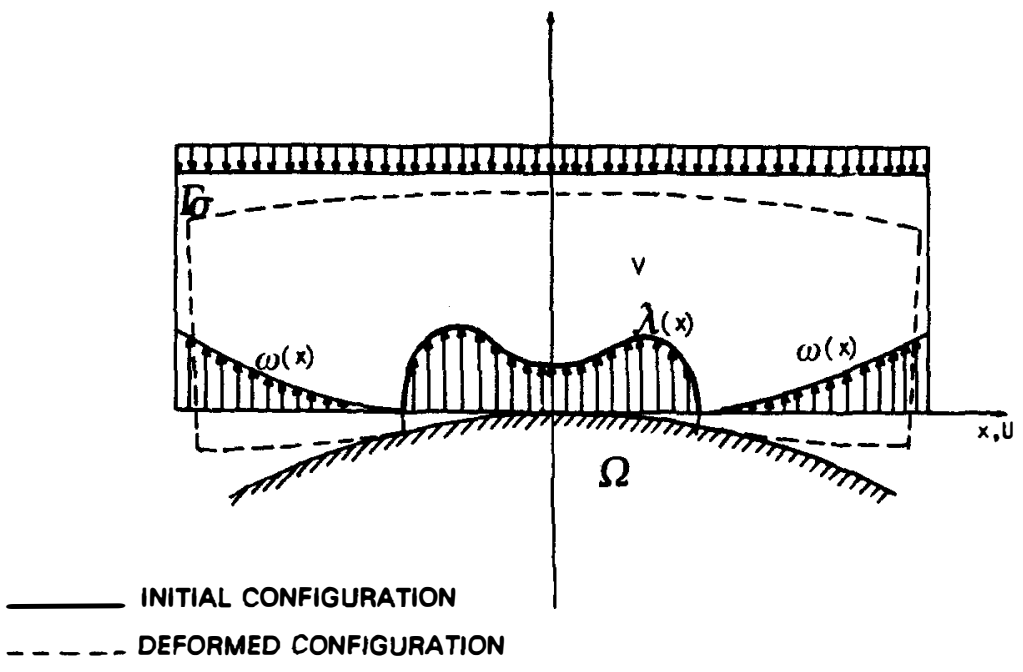

Fig. 2. 
Now, let us consider an arbitrary finite element $V_{e}$ with an interface $\Gamma_{e}$ belongs to $\Gamma_{c}$. Let $s$ the current coordinate defined on $\Gamma_{e}$. Let $q_{r e}$ be a system of nodal displacements chosen on $\Gamma_{e}$ in adopting a system of shape function $M_{e}(s)$ for $u(s)$

$$
u(s)=M_{e}(s) q_{r e}
$$

Let $N_{e}(s)$ be another system of shape function corresponding to the nodal system $\lambda_{e}$ of Lagrange's parameter $\lambda$

$$
i(s)=i_{e}^{T} N_{e}(s)
$$

According to (2.20), (2.21) and (2.22) the contact potential (2.19) of element $V_{e}$ may reduce to this matrix form

$$
\Phi_{c e}=\lambda_{e}^{T}\left(A_{e} q_{r e}+h_{e}\right)
$$

with

$$
\begin{aligned}
& h_{e}=\int N_{e} \Pi d \Gamma_{c e} \\
& A_{e}=\int N_{e}(\nabla \Pi)^{T} M_{e} \mathrm{~d} \Gamma_{c e} .
\end{aligned}
$$

The assemblage of the contact elements may be performed by using some Boolean matrices $L_{e}, G_{e}$

$$
\begin{aligned}
q_{r e} & =L_{e} q_{r} \\
\lambda_{e} & =G_{e} \lambda \\
h_{e} & =G_{e}^{T} h
\end{aligned}
$$

and writing the total contact potential as the sum of the contact potential of each element

$$
\Phi_{c}=\sum_{e} \Phi_{c e}=\lambda^{T}\left(A q_{r}+h\right)
$$

where

$$
A=\sum_{c} G_{e}^{T} A_{e} L_{e}
$$

is the constraint matrix of the contact problem. Taking the variation of $(2.28)$ with respect to $\lambda$ the problem (2.10) gives the following linear constraint in terms of the nodal displacements defined on $\Gamma_{c}$ :

$$
A q_{r} \geqslant-h
$$

\subsection{Superelement technique}

Let

$$
g_{0}=A^{T} \lambda
$$

be the generalized contact forces vector. The constraint affects only the nodal displacement defined on $\Gamma_{c}$. In order to reduce the size of the problem before the optimization process, the superelement technique is used. Application of the principle of virtual work for the entire structure

$$
\delta\left[\frac{1}{2} q^{T} K q-g^{T} g-g_{0}^{T} q_{r}-\lambda^{T} h\right]=0
$$

gives the nodal equilibrium equation

$$
\begin{aligned}
& K_{c r} q_{c}+K_{c r} q_{r}=g_{c} \\
& K_{r c} q_{c}+K_{r r} q_{r}=g_{r}+g_{0}
\end{aligned}
$$

where $q_{c}$ are the condensed displacements which must be eliminated before the optimization step

$$
q_{c}=K_{c c}^{-1}\left[g_{c}-K_{c r} q_{r}\right] .
$$

Replacing in (2.33) one obtains

$$
\bar{K}_{r r} q_{r}=\bar{g}_{r}+g_{0}
$$

with

$$
\begin{aligned}
\bar{K}_{r r} & =K_{r r}-K_{r c} K_{c c}^{-1} K_{c r} \\
\bar{g}_{r} & =g_{r}-K_{r c} K_{c c}^{-1} g_{c} .
\end{aligned}
$$

Now the size of the problem (2.32) is considerably reduced

$\delta\left[\frac{1}{2} q_{r}^{T} \bar{K}_{r r} q_{r}-\bar{g}_{r}^{T} q_{r}-\lambda\left(A q_{r}+h\right)\right]=0$

which is equivalent to the following quadratic programming problem:

minimize $\left[\frac{1}{2} q_{r}^{T} \bar{K}_{r r} q_{r}-\bar{g}_{r}^{T} q_{r}\right]$

$q_{r}$

subjected to $A q_{r} \geqslant-h$.

Once the optimal solution $q_{r}$ and the associated Lagrange parameters $i$ are known, the internal displacements and stresses of the whole structure are obtained from (2.34) by back substitution and the contact force vector from (2.31).

\subsection{Example}

It seems useful to illustrate the above finite element formulation of the contact potential $\Phi_{c}$ by taking a simple example shown by Fig. 3. If $V_{e}$ is a linear finite element having an interface $1-2$ belongs to $\Gamma_{c}$ one has the following interpolation matrix $M_{e}$ and displacement $q_{r e}$ of formulae (2.21)

$$
\begin{gathered}
M=\left|\begin{array}{cccc}
1-\eta & 0 & \eta & 0 \\
0 & 1-\eta & 0 & \eta
\end{array}\right| \quad \eta=s / l \\
q_{r e}^{T}=\left|\begin{array}{llll}
q_{11} & q_{12} & q_{21} & q_{22}
\end{array}\right|
\end{gathered}
$$

where $s$ is the current coordinate on the interface 1-2 and $l$ is its length. Iflinear interpolation is taken for $\lambda(s)$ in $(2.22)$ one has

$$
\begin{aligned}
\lambda_{e}^{r} & =\left|\begin{array}{ll}
\lambda_{1} & \lambda_{2}
\end{array}\right| \\
N_{e}(s) & =\mid \begin{array}{ll}
1-\eta & \eta
\end{array} .
\end{aligned}
$$

In these conditions the constraint matrix $A_{e}$ defined by (2.25) takes the following form:

$$
A_{e}=l\left|\begin{array}{llll}
A_{11} & A_{12} & A_{13} & A_{14} \\
A_{21} & A_{22} & A_{23} & A_{24}
\end{array}\right|
$$




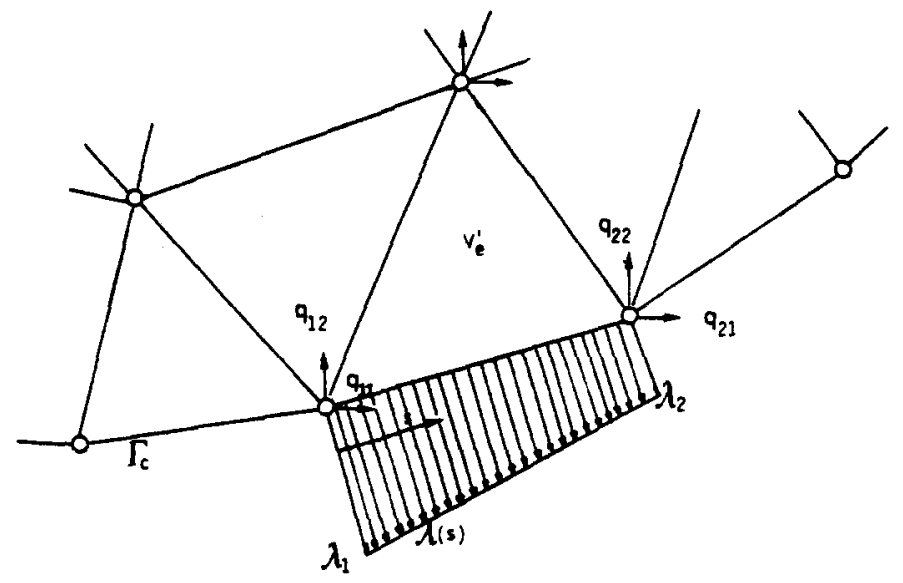

Fig. 3.

with

$$
\begin{aligned}
& A_{11}=\int_{0}^{1}(1-\eta)^{2} D_{1} \Pi(\eta) \mathrm{d} \eta \\
& A_{12}=\int_{0}^{1}(1-\eta)^{2} D_{2} \Pi(\eta) \mathrm{d} \eta \\
& A_{13}=\int_{0}^{1}(1-\eta) \eta D_{1} \Pi(\eta) \mathrm{d} \eta \\
& A_{14}=\int_{0}^{1}(1-\eta) \eta D_{2} \Pi(\eta) \mathrm{d} \eta \\
& A_{21}=\int_{0}^{1}(1-\eta) \eta D_{1} \Pi(\eta) \mathrm{d} \eta \\
& A_{22}=\int_{0}^{1}(1-\eta) \eta D_{2} \Pi(\eta) \mathrm{d} \eta \\
& A_{23}=\int_{0}^{1} \eta^{2} D_{1} \Pi(\eta) \mathrm{d} \eta \\
& A_{24}=\int_{0}^{1} \eta^{2} D_{2} \Pi(\eta) \mathrm{d} \eta .
\end{aligned}
$$

In the case of the Fig. 4 where the rigid foundation is a circle of equation

$$
\Pi\left(x_{1}, x_{2}\right)=x_{1}^{2}+x_{2}^{2}+2 x_{2} R .
$$

One has

$$
D_{1} \Pi=2 l\left(x_{11} / l+\eta\right) ; \quad D_{2} \Pi=2 R
$$

Hence

$$
A_{e}=\frac{l^{2}}{3}\left|\begin{array}{rrcr}
2\left(x_{11} / l+\frac{1}{4}\right) & 2 R / l & x_{11} / l+\frac{1}{2} & R / l \\
\left(x_{11} / l+\frac{1}{2}\right) & R / L & 2\left(x_{11} / l+\frac{3}{2}\right) & 2 R / l
\end{array}\right|
$$

and the matrix $h_{e}$ defined by $(2.24)$ is

$$
h_{e}^{T}=l^{3}\left|\frac{1}{2}\left(\frac{x_{11}}{l}\right)^{2}+\frac{1}{3} \frac{x_{11}}{l}+\frac{1}{2} \frac{1}{2}\left(\frac{x_{11}}{l}\right)^{2}+\frac{x_{11}}{3 l}+\frac{1}{4}\right| \text {. }
$$

\section{CONTACT OF TWO ELASTIC BODIES}

\subsection{Formulation}

The major difficulty for the extension of two elastic bodies consists on the fact that not only the contact zone is unknown here but the contact surface may deform during loading. We can take the boundary of one body as reference like the rigid foundation in the previous section but to do so we lost the symmetric feature of the problem. We do not treat the two body equally. For these reasons, we propose a new and general formulation described in the following. Let $V_{A}$, $V_{B}$ (Fig. 5) two elastic bodies in contact with respectively $\Gamma_{A C}, \Gamma_{B C}$ the possible contacting boundaries and $\Gamma_{A \sigma}, \Gamma_{A \sigma}$ the portion of boundaries where

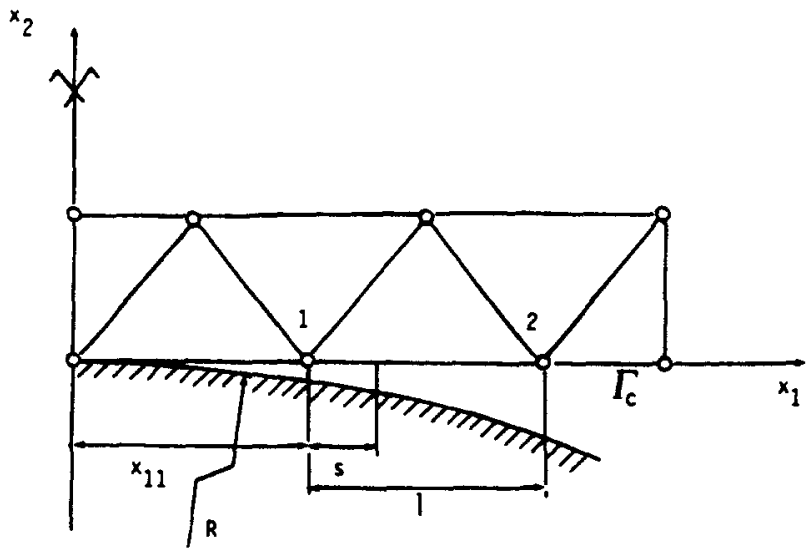

Fig. 4. 


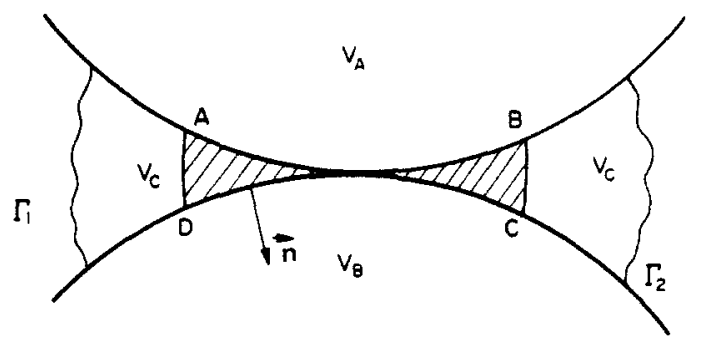

Fig. 5 .

loading $\bar{t}_{i}$ is prescribed. Let $V=V_{A} \cup V_{B}$ the total volume of the two elastic bodies and $\Gamma_{\sigma}=\Gamma_{A \sigma} \cup \Gamma_{B \sigma}$ the portion of its boundary where $\bar{t}_{i}$ are prescribed. Let $V_{C}$ is the region adjacent to the contact surface. This region has $\Gamma_{C}=\Gamma_{A C} \cup \Gamma_{B C}$ as boundary and some frontier $\Gamma=\Gamma_{1} \cup \Gamma_{2}$ which joints $\Gamma_{A C}$ and $\Gamma_{B C}$ to limit the region $V_{C}$. It may be multiple if the $V_{A}$ and $V_{B}$ are initially in contact. The basic assumption of the formulation is that the displacement field existing in $V$ may be extended in $V_{C}$ which may be considered as a fictitious material defined afterwards. Let $x_{i}$ be the Lagrangian coordinates of the material point. Under loading the configuration changes:

$$
x_{i} \rightarrow \xi_{i}=x_{i}+u_{i}
$$

and $V_{A}, V_{B}, V_{C}$ become respectively the deformed $Z_{A}$, $Z_{B}, Z_{C}$. The associated Jacobian matrix of transformation $(3.1)$ is

$$
J_{i j}=D_{i} \xi_{j}=\delta_{i j}+D_{i} u_{j}
$$

with the so-called determinant, or simply Jacobian

$$
J=\operatorname{det}\left[J_{i j}\right]
$$

The volume of the fictitious material is reduced to the value

$$
Z_{c}=\int_{V_{c}} \mathrm{~d} Z_{C}=\int_{V_{c}} J \mathrm{~d} V_{c}
$$

The narrow volume $V_{c o}$ nearly the elastic bodies and limited by $A B, C B$ is the seat of important strain and tends to zero as far as loading increases. Therefore it is evident that for a small element of volume in $V_{C O}$ the contact between $V_{A}$ and $V_{B}$ is realized when

$$
J=0
$$

otherwise, the condition of non-interpenetration would be

$$
J \geqslant 0
$$

and displacement field is called kinematically admissible in contact problem sense if it satisfies (3.6).

Now, the same formulation (2.9), (2.10) may be used here with only the following change for the dislocation potential:

$$
\phi\left(u_{i}, \lambda, \omega\right)=-\int \lambda\left(J-\omega^{2}\right) \mathrm{d} V_{c}
$$

with the new contact potential

$$
\Phi_{C}=\int \lambda J \mathrm{~d} V_{C}
$$

Noting that

$$
I_{i j}=\frac{\vec{C} J}{\vec{C} J_{i j}}
$$

is the minor of element $J_{i j}$ of the Jacobi matrix $\left[J_{i j}\right]$, one has

$$
\dot{\delta} \Phi_{\mathrm{c}}=\int i \frac{\partial J}{\partial J_{i j}} \delta J_{i j} \mathrm{~d} V_{C}
$$

and because of (3.2)

$$
\begin{aligned}
& \dot{\partial} \Phi_{C}=\int \lambda I_{i j} D_{i} \delta u_{j} \mathrm{~d} V_{C}=\int D_{i}\left(\lambda I_{i j} \delta u_{j}\right) \mathrm{d} V_{C} \\
& -\int D_{i}\left(i I_{i j}\right) \delta u_{j} \mathrm{~d} V_{C}
\end{aligned}
$$

and according to the Gauss formulae

$$
\dot{\phi} \Phi_{c}=\int i n_{i} I_{i j} \delta u_{j} \mathrm{~d} \bar{\Gamma} \times \int D_{i}\left(\lambda I_{i j}\right) \delta u_{j} \mathrm{~d} V_{C}
$$

with

$$
\bar{\Gamma}=\Gamma_{c} \cup \Gamma \text {, the total boundary of } V_{c} .
$$

Taking the variation of the modified functional

$$
\Phi^{*}\left(u_{i}, \lambda_{,} \omega\right)=\Phi\left(u_{i}\right)+\phi\left(u_{i}, \lambda, \omega\right)
$$

where $\Phi\left(u_{i}\right)$ is defined by $(2.4)$, we may find the following Euler-Lagrange's equations:

$$
\begin{array}{ll}
\text { in } V & D_{j} \sigma_{i j}+\tilde{f}_{i}=0 \\
\text { on } \Gamma_{\sigma} & n_{j} \sigma_{i j}=\tilde{t}_{i} \\
\text { on } \Gamma & n_{j} \sigma_{i j}=0 \\
\text { on } \Gamma_{C} & t_{j}=i n_{i} I_{i j} \\
\text { in } V_{C} & D_{i}\left(i I_{i j}\right)=0 .
\end{array}
$$

\subsection{Physical interpretations}

The three first equations are classical equilibrium equations. Focusing our attention on the two last new ones to give some physical interpretations. We recognize in (3.14) the presence of the normal $v_{j}$ (Fig. 6) of the strained surface $\Gamma_{C}$

$$
v_{j}=n_{i} I_{i j}
$$

so that it may be written

$$
t_{j}=\lambda v_{j}
$$

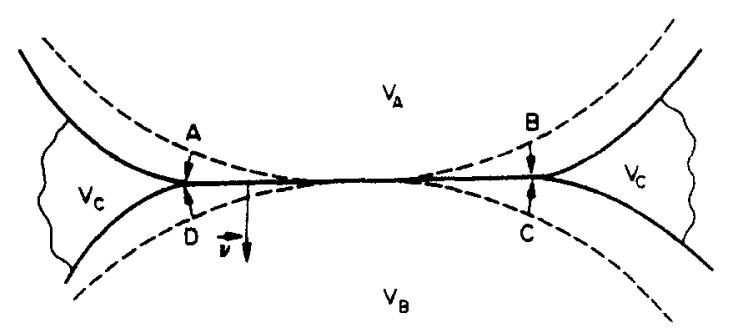

Fig. 6. 
It appears from this that the tractions are still normal to the strained surface. This is the consequence of the frictionless contact condition.

Again as in Section 3, $\lambda$ may be identified with the contact pressure on the contact surface.

Besides, in the virtual work equation (3.10) the conjugate quantity of the strain $\alpha_{i j}=D_{i} u_{j}$ is the Piola stress tensor

$$
\tau_{i j}=\lambda I_{i j}
$$

therefore, (3.14) and (3.15) may be written respectively

extermal equilibrium on $\Gamma_{C} \quad t_{j}=n_{i} \sigma_{i j}$

internal equilibrium in $V_{C} \quad D_{i} \sigma_{i j}=0$.

It is very interesting to point out that relation (3.17), (3.18) is nothing else but the normality law of an ideal blocking material defined by the potential $J$ which constitutes a dual material of the rigid perfectly plastic material [8]

$$
\tau_{i j}=\lambda \frac{\partial J}{\partial \alpha_{i j}} \quad \begin{array}{lll}
\lambda=0 & \text { if } J>0 \\
\lambda \neq 0 & \text { if } J=0 .
\end{array}
$$

3.3. Relation between the constraint function $J$ and $\Pi$

In the case of $V_{B} \equiv \Omega, \Gamma_{x c} \equiv \partial \Omega$, the body $V_{B}$ is identified to a rigid foundation examined in Section 2. It is interesting to relate constraint function $J$ defined by (3.6) to the constraint function $\Pi$ defined by (2.3).

Due to the fact that in the rigid foundation there is no strain $\Phi_{B}=0$ and the modified functional (2.9) is reduced to the form

$$
\Phi^{*}\left(u_{i}, \lambda, \omega\right)=\Phi_{A}\left(u_{i}\right)+\phi\left(u_{i}, \lambda, \omega\right)
$$

with

$$
\Phi_{A}\left(u_{i}\right)=\int U\left(\varepsilon_{i j}\right) \mathrm{d} V_{A}-\int \bar{f}_{i} u_{i} \mathrm{~d} V_{A}-\int \bar{t}_{i} u_{i} \mathrm{~d} \Gamma_{\sigma A}
$$

The dislocation potential $\phi\left(u_{i}, \lambda, \omega\right)$ is defined by (3.6) where we focus our attention to the contact potential

$$
\Phi_{c}=\int \lambda J \mathrm{~d} V_{C}
$$

Now to relate $\Pi$ of (2.19) to $J$ of (3.23) it is necessary to transform the volume integral (3.23) to a surface integral along $\Gamma_{A C} \equiv \Gamma_{C}$. Let $A \in \Gamma_{C}, B \in \delta \Omega$ be two points which must be in contact during the loading process. We introduce (Fig. 7) a special curved coordinate system

$$
\begin{aligned}
& r=r\left(x_{1}, x_{2}\right) \\
& s=s\left(x_{1}, x_{2}\right)
\end{aligned}
$$

such that for $r=r_{B}=0$, the line $r=r(B)$ coincides with the boundary of the foundation $\partial \Omega$ and the line $r=r(A)$ coincides with the boundary of the elastic solid $\Gamma_{r}$. In these conditions the frontier $\Gamma_{1}, \Gamma_{2}$ are chosen to be coincided respectively with the line $s=s_{1}$ and $s=s_{2}$. Therefore the domain $V_{c}$ of the perfect locking material discussed above is to be described

$$
V_{C} \equiv\left(0, r_{A}\right) \times\left(s_{1}, s_{2}\right)
$$

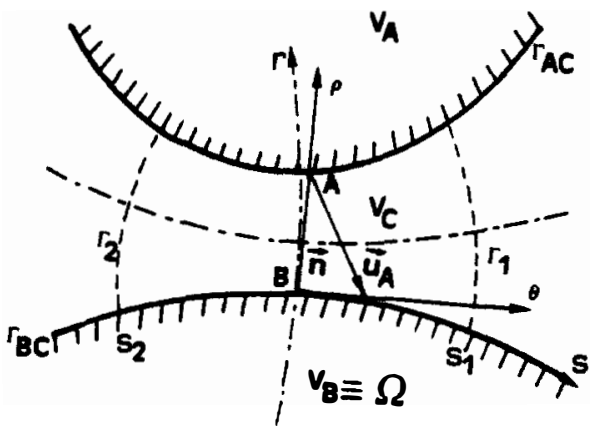

Fig. 7.

and the integral (3.22) have the more precise form

$$
\Phi_{C}=\int \lambda J d r d s .
$$

As the contact pressure $\lambda$ depends uniquely to $s$ we may write

$$
\Phi_{C}=\int_{r_{c}} \lambda \mathrm{d} s \quad \int_{0}^{r^{A}} J \mathrm{~d} r .
$$

Putting

$$
\Pi=\int_{0}^{r} J \mathrm{~d} r .
$$

It appears from (3.26) that

$$
\Phi_{c}=\int \lambda \Pi \mathrm{d} \Gamma_{c}
$$

and this is exactly the constraint potential (2.19).

As to $J$ it may be written in terms of $\alpha_{i j}$ in the following form:

$$
J=1+\alpha_{\rho \rho}+\alpha_{\theta \theta}-\alpha_{\rho \rho} \alpha_{\theta \theta}-\alpha_{\rho \theta} \alpha_{\theta \rho}
$$

where

$$
\alpha_{\rho \rho}=\frac{\partial u_{\rho}}{\partial_{p}} ; \alpha_{\theta \theta}=\frac{\partial u_{\theta}}{\partial \theta} ; \alpha_{\theta \rho}=\frac{\partial u_{\rho}}{\partial \theta} .
$$

When the solid and rigid foundation is in contact by (3.21,), we must have:

$$
\begin{aligned}
& \tau_{\rho \rho}=\lambda=\lambda \frac{\partial J}{\partial \alpha_{\rho \rho}}=\lambda\left(1+\alpha_{\theta \theta}\right) \\
& \tau_{\theta \theta}=0=\lambda \frac{\partial J}{\partial \alpha_{\theta \theta}}=\lambda\left(1+\alpha_{\rho \rho}\right) \\
& \tau_{\rho \theta}=0=\lambda \frac{\partial J}{\partial \alpha_{\rho \theta}}=-\lambda \cdot \alpha_{\theta \rho},
\end{aligned}
$$

Therefore $\alpha_{\theta \theta}=\alpha_{\theta \rho}=0$ and $\alpha_{\rho \rho}=-1$ when the contact is happened. Just before the contact, by continuity, we must expect that $\alpha_{\theta \theta}$ and $\alpha_{\rho \theta}$ are small and only the term $\alpha_{\rho \rho}$ exists in the approximated form of $J$ :

$$
J \simeq 1+\alpha_{\rho \rho} .
$$

Consequently the integral (3.27) becomes

$\Pi(\rho, u)=\rho(A)+u_{\rho}\left(\rho_{A}\right)=\rho(A)+\mathbf{n} \cdot \mathbf{u}(A)$

where $\mathbf{n}$ is the normal vector to $\partial \Omega$.

Now back to the system $x_{1}, x_{2}$ which is the Lagrangian system linked to the rigid foundation one has

$$
\Pi(\mathrm{u}=0)=\rho(A)=\rho\left(x_{1}, x_{2}\right)
$$


and due to the fact that the displacements are small

$$
\mathbf{n}=\nabla \Pi(\mathbf{u}=0)=D_{k} \Pi(\mathbf{u}=0) .
$$

According to (3.27), (3.30), (3.31) and (3.32) the noninterpenetration condition (3.6) leads to

$$
D_{k} \Pi(\mathbf{u}=0) u_{k}>-\Pi(\mathbf{u}=0)
$$

which is nothing else but the linearized inequality (2.20) already obtained in Section 2.

The unity of the two formulations has been demonstrated.

\section{NUMERICAL RESULTS}

Computing program of the rigid-elastic contact formulation exposed in Section 2 is realized by connecting the finite element algorithm ADELEF existing in our department to the mathematical programming code called ACDPAC developed by Best and Bowler [9]. The types of finite element used alternatively in the numerical examples are the isoparametric quadrangle proposed by Ergatoudis [10], Argyris and Fried [11] and the hybrid triangle proposed by Nguyen Dang Hung [12]. The latter element has the following displacement field on the interfaces

$$
\begin{aligned}
& u=\alpha_{1}+\alpha_{2} x+\alpha_{3} y+\alpha_{4} x^{2}+\alpha_{5} x y+\alpha_{6} y^{2} \\
& v=\alpha_{7}+\alpha_{8} x+\alpha_{9} y+\alpha_{10} x^{2}+\alpha_{11} x y+\alpha_{12} y^{2}
\end{aligned}
$$

and the stress field may have arbitrary degree derived from adopted general Airy's stress function

$$
F=\sum_{i=0}^{n} \sum_{j=0}^{i} \beta_{i j} x^{i-j} y^{i}
$$

This element belongs to a very efficient class of hybrid elements named the mongrel elements discussed in [13] and [14]. Externally, this element posses the same number of degree of freedom as the classical conforming quadratic triangle.

\subsection{Hertz's problem}

The classical contact problem of an infinite elastic cylinder on a rigid semi-infinite medium (Fig. 8) is tested. The analytical solution of the problem has been given early by Hertz [15]. Geometric and mechanic data of the example are

$$
\begin{gathered}
R=1000 \mathrm{~mm} \quad E=21.000 \mathrm{~kg} \mathrm{~mm}^{-2} \\
v=0.3 \quad P=600 \mathrm{~kg} \mathrm{~mm}^{-1} .
\end{gathered}
$$

Taking the advantage of the symmetry, only a half of the infinite cylinder is discretized in triangle elements. The mesh is shown successively by Figs. 8, 9, 10 and 11 . For this pattern 80 mongrel triangles are necessary with 394 degrees of freedom. The number of interface constraint elements is 16 with 36 degrees of freedom. The Fig. 12 illustrates the distribution of the pressure along the contact surface $A B$. The dotted curve is the analytical Hertz's results. The black points indicate the present results. Agreement between analytical and finite element results is satisfactory.

The CPU of this problem is $26 \mathrm{sec}$ on IBM $370-158$ at University of Liège. The Fig. 13 presents the nodal forces $g_{0}$ obtained directly in output. We may see that the coincidence with the Hertz's ellipse is more regular because the fact that the forces are relaxed by the interpolation shape function. One may distinguish the upper curve which is the forces taken from the midpoints of the contact elements and the lower curve from the connected vertex of the same elements.

\subsection{Piston rod}

Figure 1 shows how a piston rod acts on a pin under an external force $F$ distributed along $\Gamma_{\sigma}$. A clearance exists before loading and the two solids are initially in contact on the top

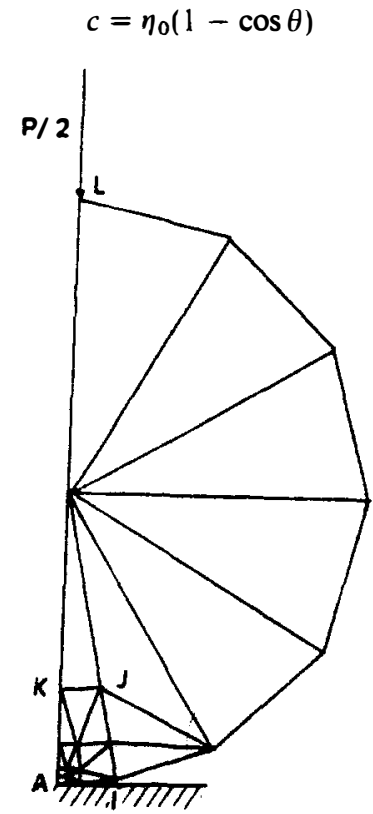

Fig. 8. Hertz's problem discretization of a semi-area of the cylinder. 


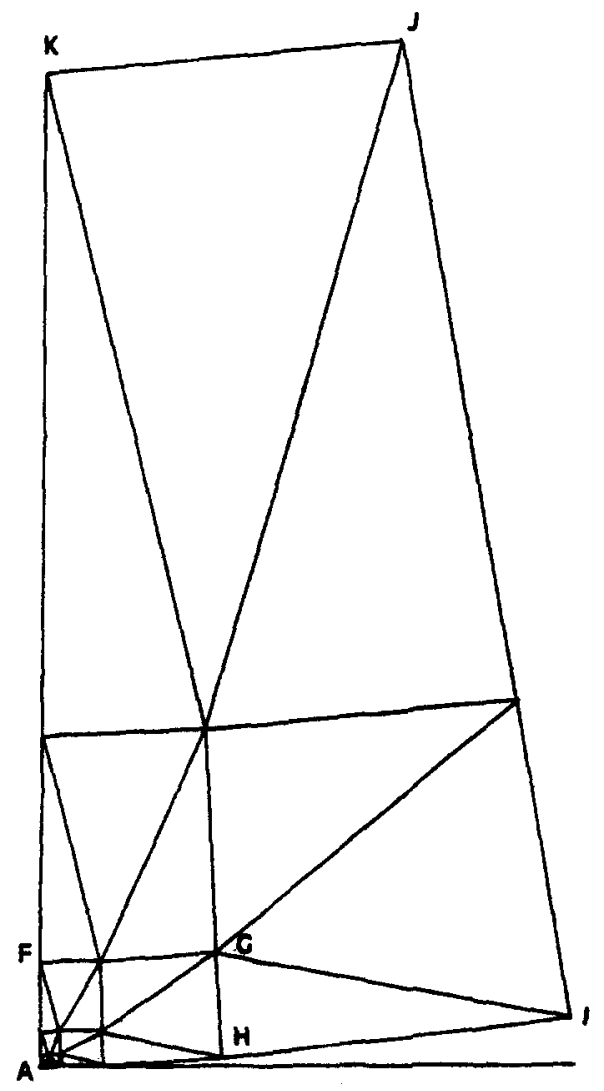

Fig. 9. Finite element mesh of region AKJI.

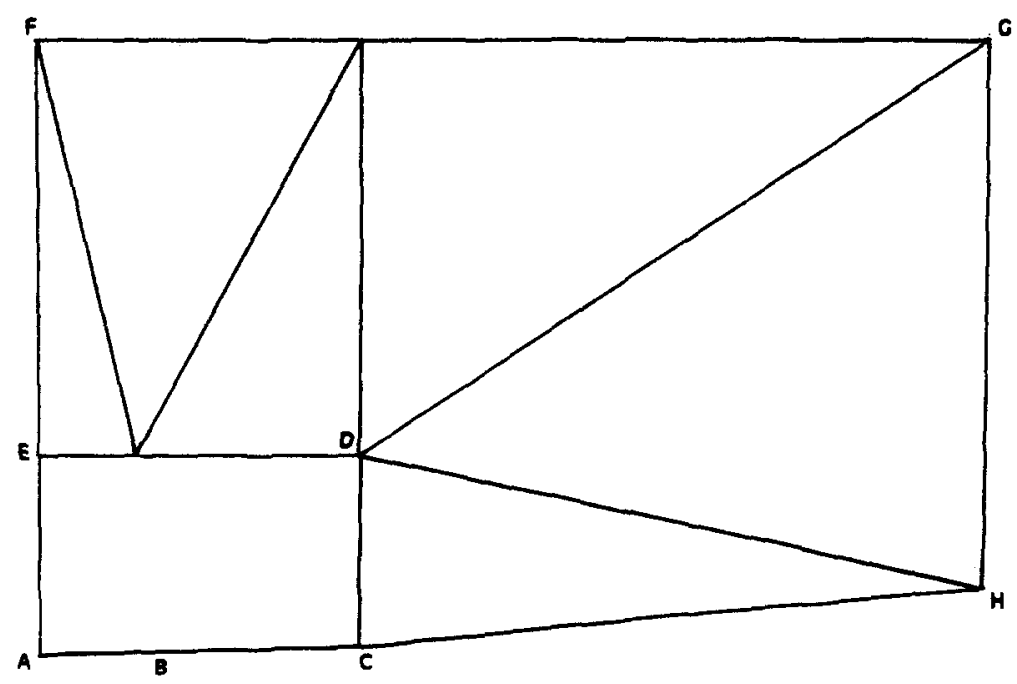

Fig. 10. Finite element mesh of region AFGH. 


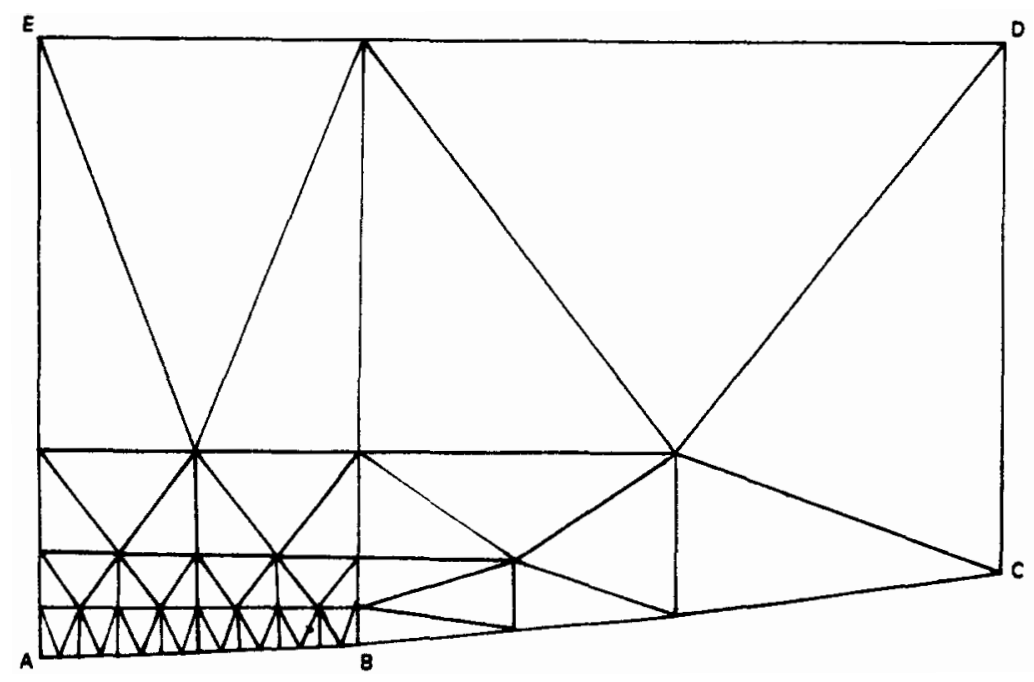

Fig. 11. Finite element mesh of region AEDC

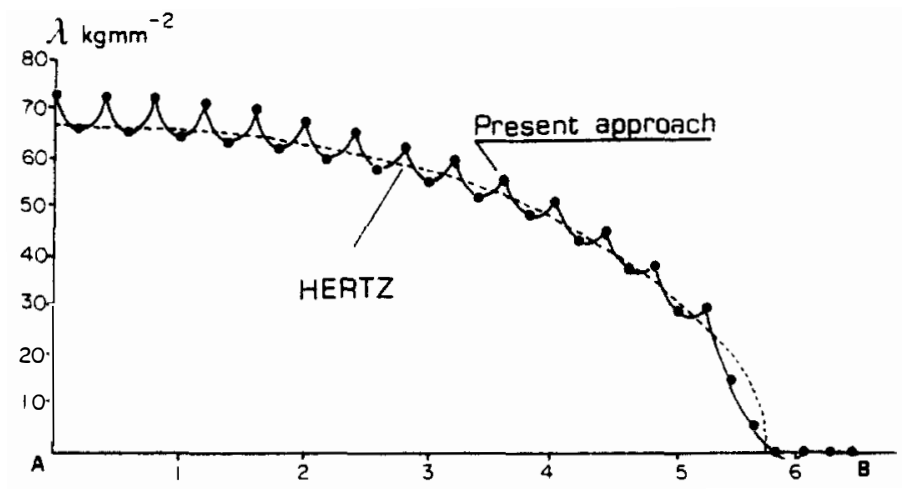

Fig. 12. Comparison of the distribution of the pressure along the contact surface.

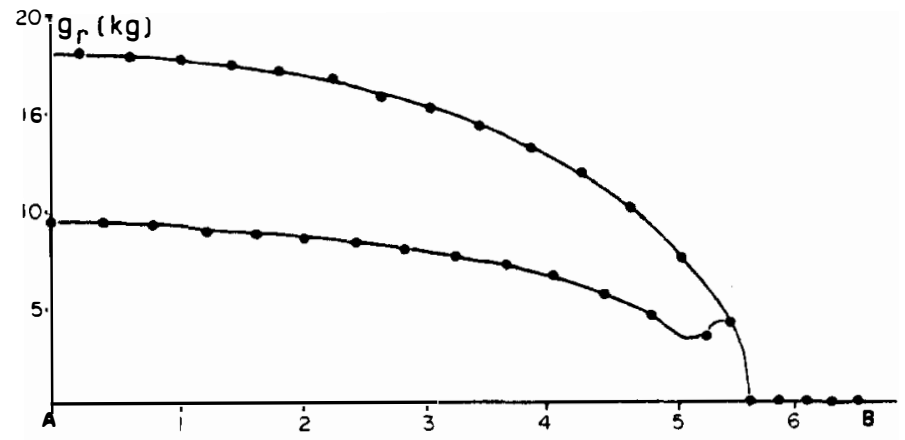

Fig. 13. distribution of the nodal forces along the contact surface. 
where

$$
\eta_{0}=R_{i}-R_{e}
$$

is the initial gap between the radius of the hole on the rod and of the pin. Following geometric data is chosen for present test:

$$
\begin{gathered}
E=7288 \mathrm{~kg} \mathrm{~mm}^{-2} \quad v=0.32 \\
t \text { (thickness) }=19.8 \mathrm{~mm} \\
R_{i}=41 \mathrm{~mm} \quad R_{\mathrm{e}}=55.5 \mathrm{~mm} \\
F=612 \mathrm{~kg} \text { (Fig. 14). }
\end{gathered}
$$

Again the symmetry allows to discretized only a half of the structure using 48 isoparametric elements. The connecting rod is considered as elastic but the shaft as rigid. The constraint function is the equation of the boundary of the pin

$$
\Pi(\xi, \eta)=\xi^{2}+\left(\eta-\eta_{0}\right)^{2}-R_{a}^{2} \geqslant 0 .
$$

The pattern (Fig. 14) needs 394 degrees of freedom with 15 contact elements corresponding to 62 degrees of freedom.

Figures 15-17 illustrate some results for the case $\eta_{0} / R_{i}=0.003$. Distributed pressures $\lambda(\theta)$ along the

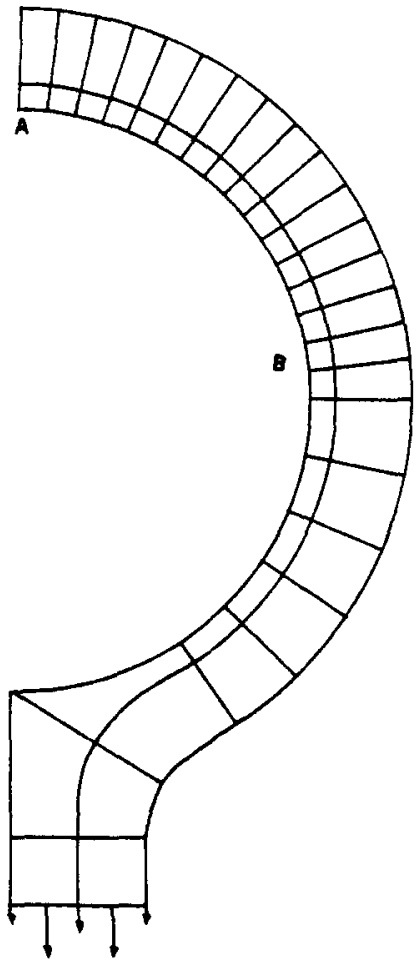

Fig. 14. Finite element mesh-the semi-area of the piston rod.

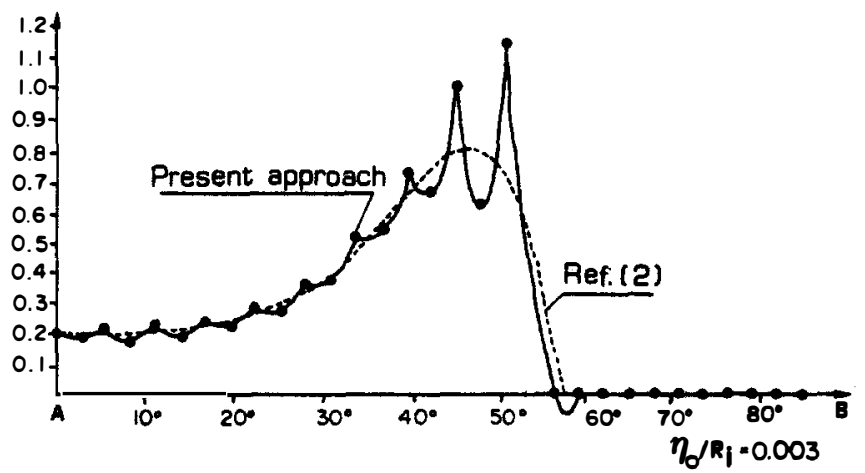

Fig. 15. Comparison of the distribution of the pressure along the contact surface.

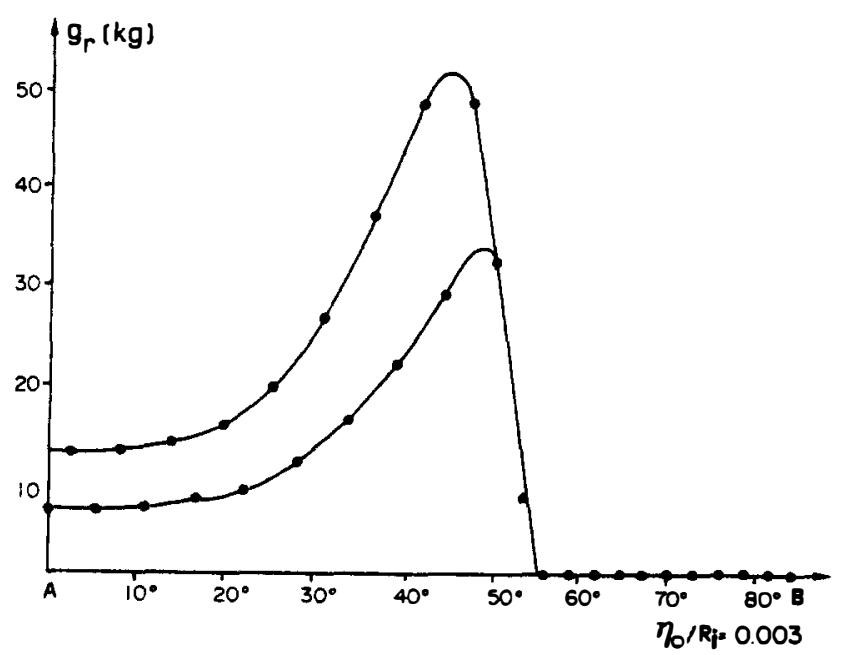

Fig. 16. Distribution of the nodal forces along the contact surface. 


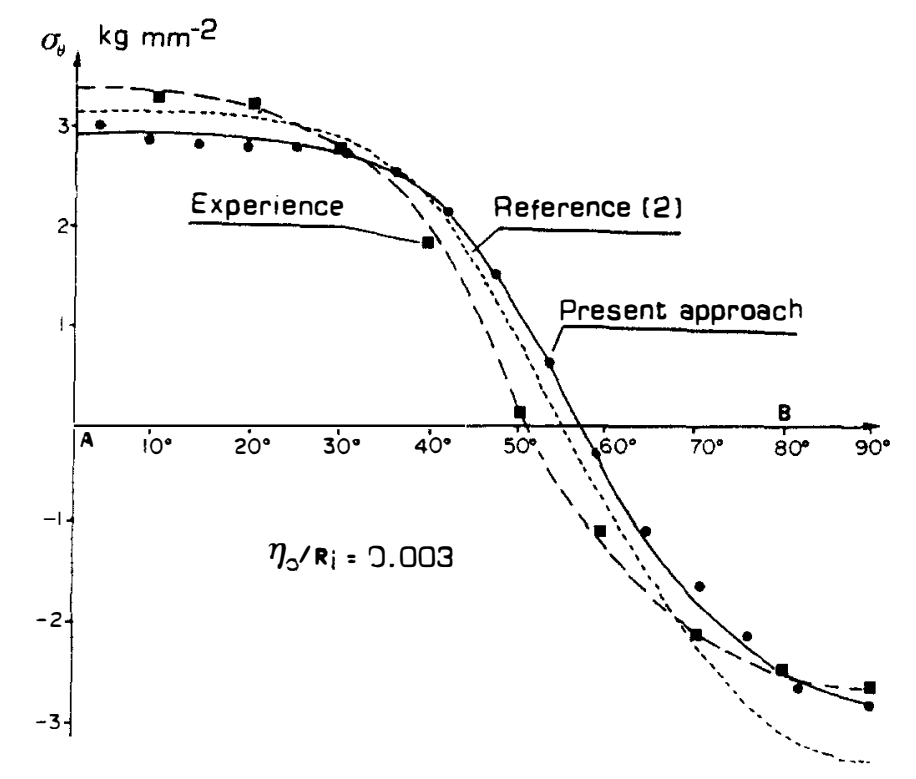

Fig. 17. Comparison of the distribution of the external circumferential stress.

angular contact and the distributed pressure of 3 cases $\eta_{0} / R_{i}=0.008, \eta_{0} / R_{i}=0.003, \eta_{0} / R_{i}=0.001$ is given by Figure 18. It is seen that for large clearance, the pressures distribution tends rapidly to elliptic form of the Hertz's analytical results.

\section{CONCLUSIONS}

Dealing with the frictionless contact problem of elastic bodies the paper presents a procedure of linearization of the non-interpenetration condition in order to realize a modelling of the contact surface into contact finite elements. This method rends the optimization problem convex and the convergence is assured. Numerical experiences for rigid-elastic contact case are very encouraging.

Extension to the elastic contact problem leads to a new concept of considering the adjacent space near the contact surfaces as a perfectly locking material. The introduction of a symmetrical non-interpenetration

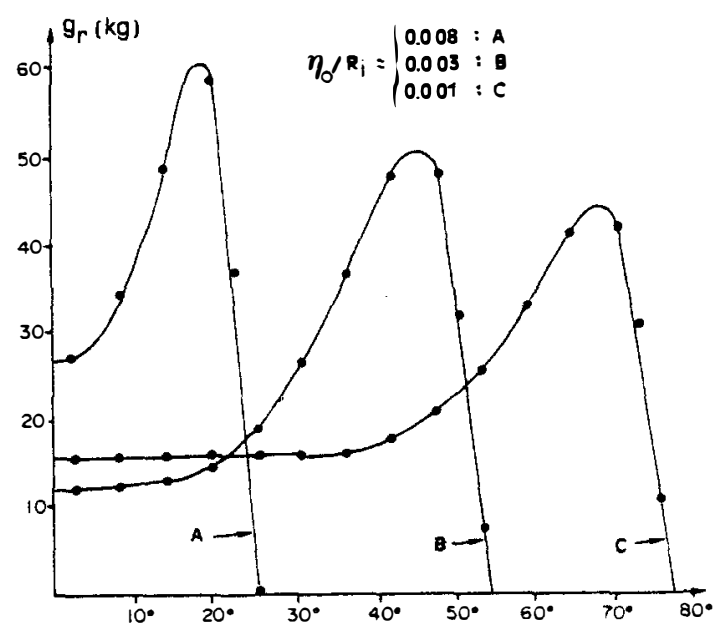

Fig. 18. Nodal forces distribution for different clearance for $F=612 \mathrm{~kg}$. condition permits to formulate elegantly the frictionless contact problem of two elastic bodies. This very promising formulation may be reduced to the rigid elastic contact problem under small displacements conditions. We hope to present in the near future the numerical results of this, and the dual formulation using equilibrium finite elements.

\section{REFERENCES}

1. S. H. Chan and I. S. Tuba, A finite element method for contact problems of solid bodies. Int. J. Mech. Sci. 13, 615-639 (1971).

2. R. Gaertner, Investigation of plane elastic contact allowing for friction. Comput. Structures 7, 59-63 (1975).

3. A. Francavilla and O. C. Zienkiewicz, A note on numerical computation of elastic contact problems. Int. J. Num. Meth. Eng. 9, 913-924 (1975).

4. B. Fredriksson, Finite element solution of surface nonlinearities in structural mechanics with special emphasis to contact and fracture mechanics problems. Comput. Structures 6, 281-290 (1976).

5. W. W. Feng and $P$. Huang, On the general contact problem of an inflated non-linear plan membrane. Int. $J$ Solids Structure 11, 437-448 (1975).

6. M. Fremond. Etude des Structures visco-élastiques stratifiées soumises á des charges harmoniques et de solides élastiques reposant sur des structures. Thèse Université de Paris (1971).

Formulation duale des énergies potentielles et complé. mentaires. Application à la méthode des éléments finis C.R. Ac. Sc. Paris, 273, série A, pp. 775-777.

7. P. D. Panagiotopoulos, $A$ nonlinear programming approach to the unilateral contact- and frictionboundary value problem in the theory of elasticity. Ingénieur-Archives 44, 421-432 (1975).

8. Nguyen Dang Hung, Modè les mathématiques et calcul numérique du comportement inélastique des matériaux. Notes de cours. Laboratoire de Mécanique des Matériaux et de Statique des Constructions, Université de Liège (1978).

9. M. J. Best and A. T. Bowler, ACDPAC, A FORTRAN IV Subroutine to solve differentiable mathematical Pro. grammes User's Guide. Level 2.0 (1976). 
10. J. G. Ergatoudis, Isoparametric elements in two and tridimensional analysis. Ph.D. Thesis Univ. Wales, Swansea (1969).

11. J. H. Argyris and I. Fried, The LUMINA element for the matrix displacement method. Aero J. ROY Aero soc. 72 , $514(1968)$

12. Nguyen Dang Hung, Direct limit analysis via rigidplastic finite elements. Com. Meth. in Appl. Mech. and Eng. 8, 81-116 (1976).

13. Nguyen Dang Hung. Sur une classe particulière des éléments finis hybrides: les éléments métis. Méthodes
Numériques dans les Sciences de IIngénieur" Proc. GAMNI Cong. 22 November to 1st December 1978, (Edited by DUNOD TECHNIQUE), pp. 53-63, Paris (1929).

14. Nguyen Dang Hung, On the monotony and the convergence of a special class of hybrid finite elements: the MONGREL Elements. IUTAM Symposium of variational method in the Mechanics. Northwestern University, Evanston, Illinois, U.S.A. 11-13 septembre 1978.

15. H. Hertz, Über die Berührung fester elastischer Körper. J. für Math. 92, 156-171 (1882). 